PROCEEDINGS OF THE

AMERICAN MATHEMATICAL SOCIETY

Volume 134, Number 9, September 2006, Pages 2729-2738

S 0002-9939(06)08281-5

Article electronically published on March 22, 2006

\title{
GAUGEABILITY FOR FEYNMAN-KAC FUNCTIONALS WITH APPLICATIONS TO SYMMETRIC $\alpha$-STABLE PROCESSES
}

\author{
MASAYOSHI TAKEDA \\ (Communicated by Richard C. Bradley)
}

\begin{abstract}
For symmetric $\alpha$-stable processes, an analytic criterion for a measure being gaugeable was obtained by Z.-Q. Chen (2002), M. Takeda (2002) and M. Takeda and T. Uemura (2004). Applying it, we consider the ultracontractivity of Feynman-Kac semigroups and expectations of the number of branches hitting closed sets in branching symmetric $\alpha$-stable processes.
\end{abstract}

\section{INTRODUCTION}

Let $\mathbb{M}^{\alpha}=\left(\mathbb{P}_{x}, X_{t}\right)(0<\alpha<2)$ be a symmetric $\alpha$-stable process on $\mathbb{R}^{d}$. Let $\mu$ be a smooth measure and $A_{t}^{\mu}$ the positive continuous additive functional (PCAF) in the Revuz correspondence to $\mu$. Then the measure $\mu$ is said to be gaugeable on an open set $D \subset \mathbb{R}^{d}$ if

$$
\sup _{x \in D} \mathbb{E}_{x}\left[\exp \left(A_{\tau_{D}}^{\mu}\right)\right]<\infty
$$

where $\tau_{D}$ is the first exit time from $D$.

For a Brownian motion, Zhao 19 introduced a class of Green-tight measures and Chen $[3$ generalized the notion of Green-tightness for more general transient Markov processes. Let $\mathbb{M}^{D}$ be the absorbing process killed upon leaving $D$ and assume that $\mathbb{M}^{D}$ is transient. Denote by $\mathcal{S}_{\infty}^{D}$ the extended class associated with $\mathbb{M}^{D}$ (see Definition 2.1 below). Chen [3] and Takeda [16] established an analytic condition for $\mu \in \mathcal{S}_{\infty}^{D}$ being gaugeable; define

$$
\lambda(\mu ; D)=\inf \left\{\mathcal{E}^{(\alpha)}(u, u): u \in C_{0}^{\infty}(D), \int_{D} u^{2}(x) \mu(d x)=1\right\} .
$$

Then the gaugeability of $\mu$ is equivalent to that of $\lambda(\mu ; D)>1$, which is also equivalent to the subcriticality of Schrödinger operators. Applying these facts, we showed in [17] the differentiability of spectral functions. The objective of this paper is to give two other applications.

Received by the editors September 17, 2004 and, in revised form, March 30, 2005.

2000 Mathematics Subject Classification. Primary 60J45, 60J40, 35J10.

Key words and phrases. Symmetric stable process, gaugeability, subcriticality, ultracontractivity, branching process.

The author was supported in part by Grant-in-Aid for Scientific Research (No.15530229 (C)(2)), Japan Society for the Promotion of Science.

(C)2006 American Mathematical Society
ublic domain 28 years from publication Reverts to public domain 28 years from publication 
The first is relevant with the ultracontractivity of Schrödinger semigroups; let $p_{t}^{\mu} f(x)=\mathbb{E}_{x}\left[\exp \left(A_{t}^{\mu}\right) f\left(X_{t}\right)\right]$ and denote by $\left\|p_{t}^{\mu}\right\|_{1, \infty}$ the operator norm of $p_{t}^{\mu}$ from $L^{1}\left(\mathbb{R}^{d}\right)$ to $L^{\infty}\left(\mathbb{R}^{d}\right)$. Then we have

Theorem 1.1. Suppose that $d>\alpha$. Let $\mu \in \mathcal{S}_{\infty}^{\mathbb{R}^{d}}$ with $\int_{\mathbb{R}^{d}} \int_{\mathbb{R}^{d}}|x-y|^{\alpha-d} d \mu(x) d \mu(y)$ $<\infty$. Then

$$
\lambda\left(\mu ; \mathbb{R}^{d}\right)>1 \Longleftrightarrow\left\|p_{t}^{\mu}\right\|_{1, \infty} \leq \frac{c}{t^{d / \alpha}}, \quad t>0 .
$$

Theorem B.1.1 in 13 says that if $2 \mu$ is gaugeable on $\mathbb{R}^{d}$, the ultracontractivity in the right-hand side of (1.2) holds. In the proof, he used the Schwartz inequality in the Feynman-Kac formula and the duality arguments, which is the reason why the gaugeability of $2 \mu$ is required. Our argument is different; a class of Girsanov transforms treated in [5] plays a crucial role.

In the second application, motivated by [10, we consider a branching symmetric $\alpha$-stable process; let $\mathbb{B}^{\alpha}=\left(\bar{X}_{t}, \overline{\mathbb{P}}_{x}\right)$ be the branching $\alpha$-symmetric stable process with the branching rate $k$, a smooth measure of $\mathbb{M}^{(\alpha)}$, and the branching mechanism $\left\{p_{n}(x)\right\}_{n \geq 2}, \sum_{2}^{\infty} p_{n}(x)=1$. Set $Q(x)=\sum_{n \geq 2} n p_{n}(x), \mu(d x)=(Q(x)-1) k(d x)$. Denote by Cap the 0 -capacity defined by the Dirichlet form generated by $\mathbb{M}^{(\alpha)}$. Then we have

Theorem 1.2. Assume that $\sup _{x \in \mathbb{R}^{d}} Q(x)<\infty$. Then for a closed set $K$ with $\operatorname{Cap}(K)>0$ and $\mu \in \mathcal{S}_{\infty}^{\mathbb{R}^{d} \backslash K}$,

$$
\lambda\left(\mu ; \mathbb{R}^{d} \backslash K\right)>1 \Longleftrightarrow \overline{\mathbb{E}}_{x}\left[N_{K}\right]<\infty .
$$

Here $N_{K}$ is the number of branches of $\mathbb{B}^{\alpha}$ ever hitting $K$.

Theorem 1.2 is an extension of [10, Theorem 4.1(iv)] to branching processes with jumps. For the proof of Theorem 1.2, we show that for $\mu \in \mathcal{S}_{\infty}^{D}$ with $\operatorname{Cap}\left(\mathbb{R}^{d} \backslash D\right)>0$

$$
\sup _{x \in D} \mathbb{E}_{x}\left[\exp \left(A_{\tau_{D}}^{\mu}\right)\right]<\infty \Longleftrightarrow \sup _{x \in D} \mathbb{E}_{x}\left[\exp \left(A_{\tau_{D}}^{\mu}\right) ; \tau_{D}<\infty\right]<\infty .
$$

In [6], the gaugeability of $\mu$ on $D$ is defined by the right-hand side of (1.4). The equation (1.4) tells us that for a measure in $\mathcal{S}_{\infty}^{D}$, two definitions of the gaugeability are equivalent.

\section{Notations AND SOME FACTS}

Let $\mathbb{M}=\left(\Omega, \mathcal{M}, \mathcal{M}_{t}, \theta_{t}, \mathbb{P}_{x}, X_{t}\right)$ be a symmetric $\alpha$-stable process on $\mathbb{R}^{d}$. Here $\left\{\mathcal{M}_{t}\right\}_{t \geq 0}$ is the minimal (augmented) admissible filtration and $\theta_{t}, t \geq 0$, are the shift operators satisfying $X_{s}\left(\theta_{t}\right)=X_{s+t}$ identically for $s, t \geq 0$. The Dirichlet form generated by $\mathbb{M}^{\alpha}$ is given by

$$
\begin{aligned}
\mathcal{E}^{(\alpha)}(u, v) & =K \iint_{\mathbb{R}^{d} \times \mathbb{R}^{d} \backslash d} \frac{(u(x)-u(y))(v(x)-v(y))}{|x-y|^{d+\alpha}} d x d y, \\
\mathcal{D}\left(\mathcal{E}^{(\alpha)}\right) & =\left\{u \in L^{2}\left(\mathbb{R}^{d}\right): \iint_{\mathbb{R}^{d} \times \mathbb{R}^{d} \backslash d} \frac{(u(x)-u(y))^{2}}{|x-y|^{d+\alpha}} d x d y<\infty\right\}
\end{aligned}
$$

$\left(K=\alpha 2^{\alpha-3} \pi^{-\frac{d+2}{2}} \sin \left(\frac{\alpha \pi}{2}\right) \Gamma\left(\frac{d+\alpha}{2}\right) \Gamma\left(\frac{\alpha}{2}\right)\right)$. Every function $u$ in $\mathcal{D}\left(\mathcal{E}^{(\alpha)}\right)$ admits a quasi-continuous version $\tilde{u}$ ([9, Theorem 2.1.3]). In the sequel we always assume that every function $u \in \mathcal{D}\left(\mathcal{E}^{(\alpha)}\right)$ is represented by its quasi-continuous version. 
Let $D \subset \mathbb{R}^{d}$ be an open set. Assume that the absorbing process $\mathbb{M}^{D}$ on $D$ is transient and let $G_{D}(x, y)$ be the Green function of $\mathbb{M}^{D}$. Note that $\mathbb{M}^{D}$ is irreducible due to the strictly positivity of the Levy measure of $\mathbb{M}$. Following [3], we make the following definition.

Definition 2.1. A positive Radon measure $\mu$ on $\mathbb{R}^{d}$ is said to be in the class $\mu \in \mathcal{S}_{\infty}^{D}$, if for any $\epsilon>0$ there exists a compact set $K \subset D$ and $\delta>0$ such that

$$
\sup _{(x, z) \in D \times D \backslash d} \int_{K^{c}} \frac{G_{D}(x, y) G_{D}(y, z)}{G_{D}(x, z)} \mu(d y) \leq \epsilon,
$$

and for all measurable sets $B \subset K$ with $\mu(B)<\delta$,

$$
\sup _{(x, z) \in D \times D \backslash d} \int_{B} \frac{G_{D}(x, y) G_{D}(y, z)}{G_{D}(x, z)} \mu(d y) \leq \epsilon .
$$

When $D=\mathbb{R}^{d}$, we remove $D$ in the notations; for example, we simply denote $\mathcal{S}_{\infty}$ for $\mathcal{S}_{\infty}^{\mathbb{R}^{d}}$. By the $3 G$-inequality, a measure $\mu$ is in $\mathcal{S}_{\infty}$ if and only if it is Green-tight in the sense of Zhao [19] (cf. [3]). For $\mu \in \mathcal{S}_{\infty}^{D}$, let $A_{t}^{\mu}$ be the positive continuous additive functional of $\mathbb{M}$ in the Revus correspondence to the measures $\mu$. It is known in [3, Proposition 2.2] that for $\mu \in \mathcal{S}_{\infty}^{D}$

$$
\sup _{x \in D} \mathbb{E}_{x}\left[A_{\tau_{D}}^{\mu}\right]=\sup _{x \in D} \int_{D} G_{D}(x, y) \mu(d y)<\infty .
$$

For a measure $\mu$ in $\mathcal{S}_{\infty}^{D}$, define

$$
\lambda(\mu ; D)=\inf \left\{\mathcal{E}^{(\alpha)}(u, u): u \in C_{0}^{\infty}(D), \int_{D} u^{2}(x) \mu(d x)=1\right\} .
$$

By Lemma 3.1 in [15] and Theorem 4.1 below, we see that $\lambda(\mu ; D)$ is the principal eigenvalue of the time changed process of $\mathbb{M}^{D}$ by $A_{\tau_{D} \wedge t}^{\mu}$. We abbreviate $\lambda\left(\mu ; \mathbb{R}^{d}\right)$ as $\lambda(\mu)$.

Let $p_{t}^{\mu, D}(x, y)$ be the integral kernel of the Feynman-Kac semigroup,

$$
\mathbb{E}_{x}\left[\exp \left(A_{t}^{\mu}\right) f\left(X_{t}\right) ; t<\tau_{D}\right]=\int_{D} p_{t}^{\mu, D}(x, y) f(y) d y,
$$

and $G^{\mu, D}(x, y)$ its Green function, $G^{\mu, D}(x, y)=\int_{0}^{\infty} p_{t}^{\mu, D}(x, y) d t$. We then have

Theorem 2.2 ([3], [16]). Let $\mu \in \mathcal{S}_{\infty}^{D}$. Then the following conditions are equivalent:

(i) $\sup _{x \in D} \mathbb{E}_{x}\left[e^{A_{\tau_{D}}^{\mu}}\right]<\infty$;

(ii) $G^{\mu, D}(x, y)<\infty$ for $x, y \in D, x \neq y$;

(iii) $\lambda(\mu ; D)>1$.

Remark 2.3. The equivalence between (i) and (iii) holds for any $\mu \in \mathcal{S}_{\infty}$ (see [16, Theorem 2.4]).

\section{Proof of Theorem 1.1}

Proof of Theorem 1.1, the $(\Longleftarrow)$ part. Let $p(t, x, y)$ be the transition probability density. Then it satisfies the upper estimate,

$$
p(t, x, y) \leq C \frac{1}{t^{d / \alpha}}\left(\frac{1}{1+\frac{|x-y|}{t^{1 / \alpha}}}\right)^{d+\alpha},
$$


where $C$ is a positive constant (see [2]). Thus by the same argument as in [1, Theorem 8.1], the integral kernel $p^{\mu}(t, x, y)$ of $P_{t}^{\mu}$ satisfies, for any $\lambda>1$,

$$
\begin{aligned}
p^{\mu}(t, x, y) & \leq C^{\prime} \frac{1}{t^{d / \alpha}}\left(\frac{1}{1+\frac{|x-y|}{t^{1 / \alpha}}}\right)^{\frac{d+\alpha}{\lambda}} \\
& \leq C^{\prime} \frac{t^{\left.\frac{d}{\alpha}+1\right) \frac{1}{\lambda}-\frac{d}{\alpha}}}{|x-y|^{\frac{d+\alpha}{\lambda}}}, \quad 0<t \leq 1,
\end{aligned}
$$

where $C^{\prime}$ is a positive constant depending on $\lambda$. In particlar, for any $x, y$ with $x \neq y$, $\sup _{0<t \leq 1} p^{\mu}(t, x, y)<\infty$. Moreover, the assumption implies that $p^{\mu}(t, x, y) \leq \frac{c}{t^{d / \alpha}}$. Therefore we have

$$
G^{\mu}(x, y) \leq \int_{0}^{1} p^{\mu}(t, x, y) d t+c \int_{1}^{\infty} \frac{1}{t^{d / \alpha}} d t<\infty .
$$

Now it follows from Theorem 2.2 that $\lambda(\mu)>1$.

To prove the converse, we need some results. Let $\mathcal{D}_{e}\left(\mathcal{E}^{(\alpha)}\right)$ be the extended Dirichlet space, that is, the family of measurable function $u$ on $\mathbb{R}^{d}$ such that $|u|<\infty$ a.e. and there exists an $\mathcal{E}^{(\alpha)}$-Cauchy sequence $\left\{u_{n}\right\}$ of functions in $\mathcal{D}\left(\mathcal{E}^{(\alpha)}\right)$ such that $\lim _{n \rightarrow \infty} u_{n}=u$ a.e. (see [9]). We denote $G \mu(x)=\int_{\mathbb{R}^{d}} G(x, y) d \mu(y)$.

Lemma 3.1. If $\mu \in \mathcal{S}_{\infty}$ satisfies $\int_{\mathbb{R}^{d}} \int_{\mathbb{R}^{d}} G(x, y) d \mu(x) d \mu(y)<\infty$, then $G \mu$ belongs to $\mathcal{D}_{e}\left(\mathcal{E}^{(\alpha)}\right)$.

Proof. First note that for $\mu \in \mathcal{S}_{\infty}$

$$
\int_{\mathbb{R}^{d}} u^{2} d \mu \leq\|G \mu\|_{\infty} \mathcal{E}^{(\alpha)}(u, u), \quad u \in \mathcal{D}_{e}\left(\mathcal{E}^{(\alpha)}\right)
$$

(cf. [14]). Then by applying (3.1) to $\mu_{K}(\cdot)=\mu(K \cap \cdot)$, we have

$$
\begin{aligned}
\int_{\mathbb{R}^{d}} \varphi d \mu_{K} & \leq(\mu(K))^{1 / 2}\left(\int_{\mathbb{R}^{d}} \varphi^{2} d \mu_{K}\right)^{1 / 2} \\
& \leq(\mu(K))^{1 / 2}\left\|G \mu_{K}\right\|_{\infty}^{1 / 2} \mathcal{E}^{(\alpha)}(\varphi, \varphi)^{1 / 2} .
\end{aligned}
$$

Hence equation (2.3) says that the measure $\mu_{K}$ is of finite energy integral, and thus

$$
\begin{aligned}
\int_{\mathbb{R}^{d}} \varphi d \mu_{K} & \leq \mathcal{E}^{(\alpha)}\left(G \mu_{K}, G \mu_{K}\right)^{1 / 2} \mathcal{E}^{(\alpha)}(\varphi, \varphi)^{1 / 2} \\
& \leq\left(\int_{\mathbb{R}^{d}} \int_{\mathbb{R}^{d}} G(x, y) d \mu_{K}(x) d \mu_{K}(y)\right)^{1 / 2} \mathcal{E}^{(\alpha)}(\varphi, \varphi)^{1 / 2} .
\end{aligned}
$$

By letting $K$ increase to $\mathbb{R}^{d}$, we find that $\mu$ is of finite energy integral, and thus $G \mu$ is in $\mathcal{D}_{e}\left(\mathcal{E}^{(\alpha)}\right)$.

Assume that $\lambda(\mu)>1$ and set

$$
h(x)=\mathbb{E}_{x}\left[e^{A_{\infty}^{\mu}}\right] .
$$

Then by Theorem 2.2. $1 \leq h(x) \leq \sup _{x} \mathbb{E}_{x}\left[e^{A_{\infty}^{\mu}}\right]<\infty$.

Lemma 3.2. Assume that $\lambda(\mu)>1$. Then it holds that

$$
h(x)=G(h \mu)(x)+1 .
$$


Proof. Define $M_{t}:=\mathbb{E}\left[\exp \left(A_{\infty}^{\mu}\right) \mid \mathcal{M}_{t}\right]$. Then by the Markov property

$$
\begin{aligned}
h\left(X_{t}\right) & =\mathbb{E}_{X_{t}}\left[\exp \left(A_{\infty}^{\mu}\right)\right]=\mathbb{E}_{x}\left[\exp \left(A_{\infty}^{\mu}\left(\theta_{t}\right)\right) \mid \mathcal{M}_{t}\right] \\
& =\mathbb{E}_{x}\left[\exp \left(A_{\infty}^{\mu}-A_{t}^{\mu}\right) \mid \mathcal{M}_{t}\right]=\exp \left(-A_{t}^{\mu}\right) M_{t},
\end{aligned}
$$

and thus

$$
\begin{aligned}
& \mathbb{E}_{x}\left[\int_{0}^{t} h\left(X_{s}\right) d A_{s}^{\mu}\right]=\mathbb{E}_{x}\left[\int_{0}^{t} \exp \left(-A_{s}^{\mu}\right) M_{s} d A_{s}^{\mu}\right] \\
& \quad=\mathbb{E}_{x}\left[M_{0}\right]-\mathbb{E}_{x}\left[\exp \left(-A_{t}^{\mu}\right) M_{t}\right]-\mathbb{E}_{x}\left[\int_{0}^{t} \exp \left(-A_{s}^{\mu}\right) d M_{s}\right] \\
& \quad=h(x)-\mathbb{E}_{x}\left[h\left(X_{t}\right)\right] .
\end{aligned}
$$

Noting that

$$
\lim _{t \rightarrow \infty} h\left(X_{t}\right)=\lim _{t \rightarrow \infty} \exp \left(-A_{t}^{\mu}\right) M_{t}=\exp \left(-A_{\infty}^{\mu}\right) \exp \left(A_{\infty}^{\mu}\right)=1
$$

we have the lemma by letting $t$ to $\infty$ in (3.2).

Suppose that $\mu \in \mathcal{S}_{\infty}$ satisfies $\int_{\mathbb{R}^{d}} \int_{\mathbb{R}^{d}} G(x, y) d \mu(x) d \mu(y)<\infty$. Then it follows from Lemma 3.1 that $G(h \mu) \in \mathcal{D}_{e}\left(\mathcal{E}^{(\alpha)}\right)$. Thus we have the Fukushima decomposition for $G(h \mu)$ ([9, Theorem 5.2.2]):

$$
G(h \mu)\left(X_{t}\right)-G(h \mu)\left(X_{0}\right)=M_{t}^{[G(h \mu)]}+N_{t}^{[G(h \mu)]} .
$$

Since the left-hand side of the above equals $h\left(X_{t}\right)-h\left(X_{0}\right)$ by Lemma 3.3 we may denote $M_{t}^{[h]}=M_{t}^{[G(h \mu)]}$. Furthermore, it follows from [9, Lemma 5.4.1] that $N_{t}^{[G(h \mu)]}=-\int_{0}^{t} h\left(X_{s}\right) d A_{s}^{\mu}$. Therefore we have

$$
h\left(X_{t}\right)-h\left(X_{0}\right)=M_{t}^{[h]}-\int_{0}^{t} h\left(X_{s}\right) d A_{s}^{\mu} .
$$

Define a martingale by

$$
M_{t}=\int_{0}^{t} \frac{1}{h\left(X_{s-}\right)} d M_{s}^{h}
$$

and denote by $L_{t}$ the unique solution of the Doleans-Dade equation:

$$
Z_{t}=1+\int_{0}^{t} Z_{s-} d M_{s}
$$

Then we see from the Doleans-Dade formula that $L_{t}$ is expressed by

$$
\begin{aligned}
L_{t} & =\exp \left(M_{t}-\frac{1}{2}\left\langle M^{c}\right\rangle_{t}\right) \prod_{0<s \leq t}\left(1+\Delta M_{s}\right) \exp \left(-\Delta M_{s}\right) \\
& =\exp \left(M_{t}-\frac{1}{2}\left\langle M^{c}\right\rangle_{t}\right) \prod_{0<s \leq t} \frac{h\left(X_{s}\right)}{h\left(X_{s-}\right)} \exp \left(1-\frac{h\left(X_{s}\right)}{h\left(X_{s-}\right)}\right) .
\end{aligned}
$$

Here $M_{t}^{c}$ is the continuous part of $M_{t}$ and $\Delta M_{s}=M_{s}-M_{s-}$. By Itô's formula applied to the semi-martingale $h\left(X_{t}\right)$ with the function $\log x$, we see that $L_{t}$ has the following expression:

$$
L_{t}=\frac{h\left(X_{t}\right)}{h\left(X_{0}\right)} \exp \left(A_{t}^{\mu}\right)
$$

Denote by $\mathbb{M}^{L}=\left(\Omega, \mathbb{P}_{x}^{L}, X_{t}\right)$ the transformed process of $\mathbb{M}^{\alpha}$ by $L_{t}, d \mathbb{P}_{x}^{L}(\omega)=$ $L_{t}(\omega) d \mathbb{P}_{x}(\omega)$. Lemma 3.1 says that $u:=\log (G(h \mu)+1) \in \mathcal{D}_{e}\left(\mathcal{E}^{(\alpha)}\right)$ and $h=\exp (u)$. 
Thus the transform by $L_{t}$ belongs to the class of Girsanov transforms considered in [5]. In particular, the Dirichlet form generated by $\mathbb{M}^{L}$ is identified as follows.

Theorem 3.3 ([5]). The transformed process $\mathbb{M}^{L}$ is an $h^{2} d x$-symmetric Hunt process and its Dirichlet form $\left(\mathcal{E}^{h}, \mathcal{F}^{h}\right)$ on $L^{2}\left(\mathbb{R}^{d}, h^{2} d x\right)$ is written as

$$
\left\{\begin{array}{l}
\mathcal{E}^{h}(u, v)=K \iint_{\mathbb{R}^{d} \times \mathbb{R}^{d} \backslash d} \frac{(u(x)-u(y))(v(x)-v(y)) h(x) h(y)}{|x-y|^{d+\alpha}} d x d y, \\
\mathcal{D}\left(\mathcal{E}^{h}\right)=\mathcal{D}\left(\mathcal{E}^{(\alpha)}\right) .
\end{array}\right.
$$

Proof of Theorem 1.1, the $(\Longrightarrow)$ part. Noting that $\left(\mathcal{E}^{h}, \mathcal{D}\left(\mathcal{E}^{h}\right)\right)$ is equivalent to $\left(\mathcal{E}^{(\alpha)}, \mathcal{D}\left(\mathcal{E}^{(\alpha)}\right)\right.$ ), we have the Sobolev inequality

$$
\left(\int_{\mathbb{R}^{d}}|f|^{\frac{2 d}{d^{-\alpha}}} h^{2} d x\right)^{\frac{d-\alpha}{2 d}} \leq c \sqrt{\mathcal{E}^{h}(f, f)}, \quad f \in \mathcal{F}^{h}
$$

and so we have by [7, Theorem 2.4.2] that

$$
\left\|p_{t}^{h} f\right\|_{\infty} \leq \frac{c}{t^{\frac{d}{\alpha}}} \int_{\mathbb{R}^{d}}|f| h^{2} d x, \quad t>0 .
$$

Here $p_{t}^{h}$ is the semigroup of $\mathbb{M}^{L}$. We see from (3.5) that

$$
p_{t}^{h} f(x)=\frac{1}{h(x)} p_{t}^{\mu}(h f)(x) .
$$

Hence

$$
\begin{aligned}
\left\|p_{t}^{\mu} f\right\|_{\infty} & =\left\|h p_{t}^{h}\left(\frac{|f|}{h}\right)\right\|_{\infty} \leq \frac{c\|h\|_{\infty}}{t^{\frac{d}{\alpha}}} \int_{\mathbb{R}^{d}} \frac{|f|}{h} h^{2} d x \\
& \leq \frac{c\|h\|_{\infty}^{2}}{t^{\frac{d}{\alpha}}} \int_{\mathbb{R}^{d}}|f| d x,
\end{aligned}
$$

which implies

$$
\left\|p_{t}^{\mu}\right\|_{1, \infty} \leq \frac{c^{\prime}}{t^{\frac{d}{\alpha}}}, \quad t>0
$$

Example 3.4. Let $\sigma_{r}$ be the surface measure of the sphere $\partial B_{r}=\{|x|=r\}$. Since the symmetric $\alpha$-stable process hits the sphere $\partial B_{r}$ if $1<\alpha$, the measure $\sigma_{r}$ is in $\mathcal{S}_{\infty}$. Combining Theorem 1.1 with [18, Example 4.1], we find that

$$
\begin{aligned}
\left\|p_{t}^{\sigma_{r}}\right\|_{1, \infty} \leq \frac{c}{t^{\frac{d}{\alpha}}}, \quad t>0 & \Longleftrightarrow \lambda\left(\sigma_{r}\right)>1 \\
& \Longleftrightarrow\left\{\frac{\sqrt{\pi} \Gamma\left(\frac{d+\alpha}{2}-1\right) \Gamma\left(\frac{\alpha}{2}\right)}{\Gamma\left(\frac{\alpha-1}{2}\right) \Gamma\left(\frac{d-\alpha}{2}\right)}\right\}^{\frac{1}{\alpha-1}}>r .
\end{aligned}
$$

Here $\Gamma(x)=\int_{0}^{\infty} e^{-t} t^{x-1} d t$. 


\section{Proof of Theorem 1.2}

Let $K$ be a closed set with $\operatorname{Cap}(K)>0$ and denote $D=\mathbb{R}^{d} \backslash K$. Let $\left(\mathcal{E}_{D}^{(\alpha)}, \mathcal{D}\left(\mathcal{E}_{D}^{(\alpha)}\right)\right)$ be the Dirichlet form generated by the absorbing process of $\mathbb{M}^{(\alpha)}$ on $D$ (cf. Section 4.4 in [9]). Let $\mathcal{D}_{e}\left(\mathcal{E}_{D}^{(\alpha)}\right)$ be the extended Dirichlet space of $\left(\mathcal{E}_{D}^{(\alpha)}, \mathcal{D}\left(\mathcal{E}_{D}^{(\alpha)}\right)\right)$. Because of the remark just below Definition 3.1 in [3] and Proposition 2.3 in [3], we have the next theorem in the same manner as in [17, Theorem 3.3].

Theorem 4.1. Let $\mu \in \mathcal{S}_{\infty}^{D}$. Then the embedding of $\left(\mathcal{E}_{D}^{(\alpha)}, \mathcal{D}_{e}\left(\mathcal{E}_{D}^{(\alpha)}\right)\right)$ to $L^{2}(D ; \mu)$ is compact.

Moreover, by the same argument as in [17, Section 4], we have

Lemma 4.2. If $\lambda(\mu ; D)=1$, then there exists a positive continuous bounded function $u_{0}$ on $D$ such that

$$
u_{0}(x)=\mathbb{E}_{x}\left[\int_{0}^{\tau_{D}} u_{0}\left(X_{t}\right) d A_{t}^{\mu}\right]
$$

and so equation (4.1) says that the function $u_{0}$ is $p_{t}^{\mu, D}$-excessive, $p_{t}^{\mu, D} u_{0}(x) \leq u_{0}(x)$ for every $t>0$ and $x \in D$.

Set

$$
h_{D}(x)=\mathbb{E}_{x}\left[\exp \left(A_{\tau_{D}}^{\mu}\right) ; \tau_{D}<\infty\right] .
$$

Lemma 4.3. If $\sup _{x \in D} h_{D}(x)<\infty$, then $h_{D}$ is a $p_{t}^{\mu, D}$-excessive function. Moreover, it satisfies

$$
h_{D}(x)=\mathbb{P}_{x}\left[\tau_{D}<\infty\right]+\mathbb{E}_{x}\left[\int_{0}^{\tau_{D}} h_{D}\left(X_{t}\right) d A_{t}^{\mu}\right] .
$$

Proof. The $p_{t}^{\mu, D}$-excessiveness follows from the Markov property

$$
\begin{aligned}
p_{t}^{\mu, D} h_{D}(x) & =\mathbb{E}_{x}\left[\exp \left(A_{t}^{\mu}\right) \mathbb{E}_{X_{t}}\left[\exp \left(A_{\tau_{D}}^{\mu}\right) ; \tau_{D}<\infty\right] ; t<\tau_{D}\right] \\
& =\mathbb{E}_{x}\left[\mathbb{E}_{x}\left[\exp \left(A_{t}^{\mu}+A_{\tau_{D}}^{\mu}\left(\theta_{t}\right)\right) ; \tau_{D}\left(\theta_{t}\right)<\infty, t<\tau_{D} \mid \mathcal{M}_{t}\right]\right. \\
& =\mathbb{E}_{x}\left[\exp \left(A_{\tau_{D}}^{\mu}\right) ; t<\tau_{D}<\infty\right] \leq h_{D}(x) .
\end{aligned}
$$

For the proof of (4.2), note that

$$
\begin{aligned}
\mathbb{E}_{x} & {\left[\int_{0}^{\tau_{D}} h_{D}\left(X_{t}\right) d A_{t}^{\mu}\right]=\mathbb{E}_{x}\left[\int_{0}^{\tau_{D}} \mathbb{E}_{X_{t}}\left[\exp \left(A_{\tau_{D}}^{\mu}\right) ; \tau_{D}<\infty\right] d A_{t}^{\mu}\right] } \\
& =\mathbb{E}_{x}\left[\int_{0}^{\tau_{D}} \mathbb{E}_{x}\left[\exp \left(A_{\tau_{D}}^{\mu}\left(\theta_{t}\right)\right) ; \tau_{D}\left(\theta_{t}\right)<\infty \mid \mathcal{M}_{t}\right] d A_{t}^{\mu}\right] \\
& =\mathbb{E}_{x}\left[\int_{0}^{\tau_{D}} \exp \left(-A_{t}^{\mu}\right) \mathbb{E}_{x}\left[\exp \left(A_{\tau_{D}}^{\mu}\right) ; t<\tau_{D}<\infty \mid \mathcal{M}_{t}\right] d A_{t}^{\mu}\right] .
\end{aligned}
$$

Set

$$
\begin{aligned}
X_{t} & =\exp \left(-A_{t}^{\mu}\right) \exp \left(A_{\tau_{D}}^{\mu}\right) I_{\left\{t<\tau_{D}<\infty\right\}}, \\
Y_{t} & =\exp \left(-A_{t}^{\mu}\right) \mathbb{E}_{x}\left[\exp \left(A_{\tau_{D}}^{\mu}\right) \mid \mathcal{M}_{t}\right] I_{\left\{t<\tau_{D}<\infty\right\}} .
\end{aligned}
$$

Then applying Exercise (1.13) of Chap. V in [11, we have

$$
\mathbb{E}_{x}\left[\int_{0}^{\infty} X_{t} d A_{t}^{\mu}\right]=\mathbb{E}_{x}\left[\int_{0}^{\infty} Y_{t} d A_{t}^{\mu}\right] .
$$


Namely, the right-hand side of (4.3) equals

$$
\begin{aligned}
& \mathbb{E}_{x}\left[\exp \left(A_{\tau_{D}}^{\mu}\right) \int_{0}^{\tau_{D}} \exp \left(-A_{t}^{\mu}\right) d A_{t}^{\mu}\right] \\
= & \mathbb{E}_{x}\left[\exp \left(A_{\tau_{D}}^{\mu}\right) ; \tau_{D}<\infty\right]-\mathbb{P}_{x}\left[\tau_{D}<\infty\right],
\end{aligned}
$$

which implies (4.2).

Lemma 4.4. If $\sup _{x \in D} h_{D}(x)<\infty$, then $\lambda(\mu ; D) \geq 1$.

Proof. Suppose that $\lambda(\mu ; D)<1$. Then there is $0<\theta<1$ such that $\lambda(\theta \mu ; D)=1$. It follows from Lemma 4.3 that $h$-tranformation $p_{t}(x, y):=\frac{1}{h_{D}(x)} p_{t}^{\mu, D}(x, y) h_{D}(y)$ is a transition probability density. Denote by $\mathbb{M}$ the Markov processes generated by $p_{t}(x, y)$. The kernel $p_{t}^{\theta}(x, y):=\frac{1}{h_{D}(x)} p_{t}^{\theta \mu, D}(x, y) h_{D}(y)$ is also a transition probability density. In fact, $p_{t}^{\theta}(x, y)$ is the transition density of the subprocess of $\mathbb{M}$ by the multiplicative functional $\exp \left(-(1-\theta) A_{t}^{\mu}\right)$;

$$
\frac{1}{h_{D}(x)} p_{t}^{\theta \mu, D}\left(h_{D} f\right)(x)=\frac{1}{h_{D}(x)} \mathbb{E}_{x}\left[\exp \left(-(1-\theta) A_{t}^{\mu}\right) \exp \left(A_{t}^{\mu}\right) h_{D}\left(X_{t}\right) f\left(X_{t}\right) ; t<\tau_{D}\right] .
$$

Hence $p_{t}^{\theta}(x, y)$ defines a transient Markov process, which is contradictory to Theorem 2.2

Lemma 4.5. If $\sup _{x \in D} h_{D}(x)<\infty$, then $\lambda(\mu ; D)>1$.

Proof. Suppose that $\lambda(\mu ; D)=1$. Let $u_{0}$ be the function in Lemma 4.2. Then Theorem 2.2 says that $\frac{1}{u_{0}(x)} p_{t}^{\mu, D}(x, y) u_{0}(y)$ is a transition probability density generating a recurrent Markov process. Moreover, since

$$
\frac{1}{u_{0}(x)} \mathbb{E}_{x}\left[\exp \left(A_{t}^{\mu}\right) u_{0}\left(X_{t}\right)\left(\frac{h_{D}}{u_{0}}\right)\left(X_{t}\right) ; t<\tau_{D}\right] \leq \frac{h_{D}}{u_{0}}(x),
$$

that is, $h_{D} / u_{0}$ is excessive with respect to $\frac{1}{u_{0}(x)} p_{t}^{\mu, D}(x, y) u_{0}(y)$, there exists a positive constant $c$ such that $h_{D}=c u_{0}$. However, this is impossible by Lemma 4.2 and Lemma 4.3 because $\mathbb{P}_{x}\left[\tau_{D}<\infty\right]>0$ for all $x \in D$. Therefore, Lemma 4.4 leads us to this lemma.

By combining Lemma 4.5 and Theorem 2.2, we have

Theorem 4.6. Let $\mu \in \mathcal{S}_{\infty}^{D}$. Then

$$
\begin{aligned}
\lambda(\mu ; D)>1 & \Longleftrightarrow \sup _{x \in D} \mathbb{E}_{x}\left[\exp \left(A_{\tau_{D}}^{\mu}\right)\right]<\infty \\
& \Longleftrightarrow \sup _{x \in D} \mathbb{E}_{x}\left[\exp \left(A_{\tau_{D}}^{\mu}\right) ; \tau_{D}<\infty\right]<\infty .
\end{aligned}
$$

Proof of Theorem 1.2. Let $\bar{X}_{t}^{D}$ be the process that stops when $\bar{X}_{t}$ reaches $K$. Then, $N_{K}$ is the number of the offsprings of $\bar{X}_{t}^{D}$ that reach $K$. Let $N_{t}$ be the number of the offspring of $\bar{X}_{t}^{D}$ at $t$ and $N_{t}^{D}$ the number of the offspring of $\bar{X}_{t}^{D}$ that stay in $D$ at $t$. Then by the strong Markov property, $m(t, x):=\overline{\mathbb{E}}_{x}\left[N_{t}\right]$ and $m^{D}(t, x):=\overline{\mathbb{E}}_{x}\left[N_{t}^{D}\right]$ satisfy

$$
m(t, x)=\mathbb{E}_{x}\left[\int_{0}^{\sigma_{K} \wedge t} e^{-A_{s}^{k}} m\left(t-s, X_{s}\right) Q\left(X_{s}\right) d A_{s}^{k}\right]+\mathbb{E}_{x}\left[e^{-A_{\sigma_{K} \wedge t}^{k}}\right]
$$


and

$$
m^{D}(t, x)=\mathbb{E}_{x}\left[\int_{0}^{\sigma_{K} \wedge t} e^{-A_{s}^{k}} m^{D}\left(t-s, X_{s}\right) Q\left(X_{s}\right) d A_{s}^{k}\right]+\mathbb{E}_{x}\left[e^{-A_{t}^{k}} ; t<\sigma_{K}\right],
$$

respectively. Moreover, functions

$$
v(t, x):=\mathbb{E}_{x}\left[\exp \left(A_{\sigma_{K} \wedge t}^{\mu}\right)\right], \quad v^{D}(t, x):=\mathbb{E}_{x}\left[\exp \left(A_{t}^{\mu}\right) ; t<\sigma_{K}\right]
$$

also satisfy the equations above, respectively. Since the bounded solution of each equation above is unique by [8, Lemma 4.4.2, Lemma 4.3.1], we see that

$$
\overline{\mathbb{E}}_{x}\left[N_{t}\right]=\mathbb{E}_{x}\left[\exp \left(A_{\sigma_{K} \wedge t}^{\mu}\right)\right], \quad \overline{\mathbb{E}}_{x}\left[N_{t}^{D}\right]=\mathbb{E}_{x}\left[\exp \left(A_{t}^{\mu}\right) ; t<\sigma_{K}\right]
$$

and

$$
\begin{aligned}
\overline{\mathbb{E}}_{x}\left[N_{K}\right] & =\lim _{t \rightarrow \infty}\left(\overline{\mathbb{E}}_{x}\left[N_{t}\right]-\overline{\mathbb{E}}_{x}\left[N_{t}^{D}\right]\right) \\
& =\mathbb{E}_{x}\left[\exp \left(A_{\sigma_{K}}^{\mu}\right) ; \sigma_{K}<\infty\right] .
\end{aligned}
$$

Now Theorem 4.6 leads us to Theorem 1.2.

Lemma 4.7. Let $K$ be a closed set with $\operatorname{Cap}(K)>0$. Then for $\mu \in \mathcal{S}_{\infty}^{D}, \lambda(\mu ; D)>$ $\lambda(\mu)$.

Proof. Let $F$ be the topological support of $\mu$ and denote by $\left(\check{\mathcal{E}}^{(\alpha)}, \mathcal{D}\left(\check{\mathcal{E}}^{(\alpha)}\right)\right)$ the Dirichlet form on $L^{2}(F, \mu)$ generated by the time changed process by $A_{t}^{\mu}$. By (3.1) the restriction of $u \in \mathcal{D}_{e}\left(\mathcal{E}^{(\alpha)}\right)$ to $F,\left.u\right|_{F}$, belongs to $\mathcal{D}\left(\tilde{\mathcal{E}}^{(\alpha)}\right)$ (9, Lemma 6.2.2]), and

$$
\mathcal{E}^{(\alpha)}(u, u)=\check{\mathcal{E}}^{(\alpha)}\left(\left.u\right|_{F},\left.u\right|_{F}\right) .
$$

As a result of Theorem 4.1, the embedding of $\left(\mathcal{E}_{D}^{(\alpha)}, \mathcal{D}_{e}\left(\mathcal{E}_{D}^{(\alpha)}\right)\right)$ to $L^{2}(D ; \mu)$ is compact. Hence there exists the function which attains the infimum in the definition of $\lambda(\mu ; D)$. Consequently, $\lambda(\mu ; D) \geq \lambda(\mu)$. Indeed, suppose that $\lambda(\mu ; D)=\lambda(\mu)$ and let $u_{0}$ and $u_{D}$ be functions attaining $\lambda(\mu)$ and $\lambda(\mu ; D)$, respectively. Then $\left.u_{0}\right|_{F}=\left.u_{D}\right|_{F} \mu$-a.e. because $\left.u_{0}\right|_{F}$ and $\left.u_{D}\right|_{F}$ are normalized principal eigenfunctions of the time changed process by $A_{t}^{\mu}$ and it is irreducible.

Since for any $\varphi \in \mathcal{D}_{e}\left(\mathcal{E}^{(\alpha)}\right)$

$$
\mathcal{E}^{(\alpha)}\left(u_{0}, \varphi\right)=\int_{\mathbb{R}^{d}} u_{0} \varphi d \mu, \quad \mathcal{E}^{(\alpha)}\left(u_{D}, \varphi\right)=\int_{\mathbb{R}^{d}} u_{D} \varphi d \mu,
$$

we have $\mathcal{E}^{(\alpha)}\left(u_{0}-u_{D}, \varphi\right)=0$ for any $\varphi \in \mathcal{D}_{e}\left(\mathcal{E}^{(\alpha)}\right)$, Therefore $u_{0}=u_{D}$, which contradicts that $\operatorname{Cap}(K)>0$.

Corollary 4.8. Let $K$ be a closed set with $\operatorname{Cap}(K)>0$. If $\lambda(\mu) \geq 1$ and $\mu \in \mathcal{S}_{\infty}^{D}$, then $\overline{\mathbb{E}}_{x}\left[N_{K}\right]<\infty$.

Example 4.9. Suppose that $d=1$ and $1<\alpha<2$. Let $k=\delta_{a}, a \neq 0$, the Dirac measure at $a$ and $p_{2}(x)=1$. Then it is known in [12] that

$$
\lambda\left(\delta_{a} ; \mathbb{R} \backslash\{0\}\right)=-\frac{\Gamma(\alpha) \cos \left(\frac{\pi \alpha}{2}\right)}{2|a|^{\alpha-1}} .
$$

Hence

$$
\sup _{x \in \mathbb{R} \backslash\{0\}} \overline{\mathbb{E}}\left[N_{\{0\}}\right]<\infty \Longleftrightarrow 0<|a|<\left(-\frac{\Gamma(\alpha) \cos \left(\frac{\pi \alpha}{2}\right)}{2}\right)^{1 /(\alpha-1)} .
$$




\section{REFERENCES}

1. S. Albeverio, P. Blanchard and Z.M. Ma, Feynman-Kac semigroups in terms of signed smooth measures, Random Partial Differential Equations, eds: U. Hornung et al., Birkhäuser, 1991 MR1185735 (93i:60140)

2. A. Bendikov, Asymptotic formulas for symmetric stable semigroups, Expo. Math., 12 (1994), 381-384. MR1297844 (95j:60029)

3. Z.-Q. Chen, Gaugeability and Conditional Gaugeability, Trans. Amer. Math. Soc., 354 (2002), 4639-4679. MR 1926893 (2003i:60127)

4. Z.-Q. Chen and R.M. Song, General gauge and conditional gauge theorems, Ann. Probab., 30 (2002), 1313-1339. MR1920109 (2003f:60135)

5. Z.-Q. Chen and S.T. Zhang, Girsanov and Feynman-Kac type transformations for symmetric Markov processes, Ann. Inst. Henri Poincare, 38 (2002), 475-505. MR 1914937 (2004e:60128)

6. K.L. Chung and Z. Zhao, From Brownian Motion to Schrödinger's Equation, Springer (1995). MR.1329992 (96f:60140)

7. E.B. Davies, Heat Kernels and Spectral Theory, Cambridge Univ. Press, Cambridge, U.K. (1989). MR0990239 (90e:35123)

8. D.A. Dawson, Measure-valued Markov processes, Lectures Notes in Math., 1541, Springer, (1993), 1-260. MR.1242575 (94m:60101)

9. M. Fukushima, Y. Oshima and M. Takeda, Dirichlet Forms and Symmetric Markov Processes, De Gruyter, 1994. MR1303354(96f:60126)

10. A. Grigor'yan and M. Kelbert, Recurrence and transience of branching diffusion processes on Riemannian manifolds, Ann. Probab., 31 (2003), 244-284. MR.1959793 (2003k:60211)

11. D. Revuz and M. Yor, Continuous martingales and Brownian motion, Third edition, Grundlehren der Mathematischen Wissenschaften, 293, Springer, 1999. MR1725357 (2000h:60050)

12. Y. Shiozawa, Principal eigenvalues for time changed processes of one-dimensional $\alpha$-stable processes, Probability and Mathematical Statistics, 24 (2004), 111-122.

13. B. Simon, Schrödinger semigroups, Bull. Amer. Math. Soc., 7, 447-536, (1982). MR0670130 (86b:81001a)

14. P. Stollmann and J. Voigt, Perturbation of Dirichlet forms by measures, Potential Analysis, 5 (1996) 109-138. MR1378151 (97e:47065)

15. M. Takeda, Exponential decay of lifetime and a Theorem of Kac on total occupation times, Potential Analysis, 11 (1999), 235-247. MR1717103 (2000i:60084)

16. M. Takeda, Subcriticality and conditional gaugeability of generalized Schrödinger operators, J. Funct. Anal., 191 (2002), 343-376. MR.1911190 (2003e:60176)

17. M. Takeda and K. Tsuchida, Differentiability of spectral functions for symmetric $\alpha$-stable process, to appear in Trans. Amer. Math. Soc.

18. M. Takeda and T. Uemura, Subcriticality and gaugeability for symmetric $\alpha$-stable processes, Forum Math. 16 (2004), 505-517. MR2044025 (2005d:60124)

19. Z. Zhao, Subcriticality and gaugeability of the Schrödinger operator, Trans. Amer. Math. Soc., 334 (1992), 75-96. MR1068934 (93a:81041)

Mathematical Institute, Tohoku University, Aoba, Sendai, 980-8578, Japan

E-mail address: takeda@math.tohoku.ac.jp 